\title{
Functional definition of regions necessary for replication and incompatibility in the Mycobacterium fortuitum plasmid pAL5000
}

\author{
Pelle Stolt and Neil G. Stoker \\ Author for correspondence: Pelle Stolt. Tel: +44171 927 2425. Fax: +44 1716374314. \\ e-mail: p.stolt@lshtm.ac.uk
}

Department of Clinical Sciences, Bacterial Molecular Genetics Unit, London School of Hygiene \& Tropical Medicine, Keppel Street, London WC1E 7HT, UK

\begin{abstract}
Different parts of the Mycobacterium fortuitum plasmid pAL5000 necessary for plasmid replication and incompatibility were defined and studied. Two ORFs, named repA and repB, were defined which are necessary for replication. A pAL5000 derivative deleted in these genes can be made to replicate by providing the gene products in trans. A 435 bp fragment was defined which was necessary in cis for replication and which had an influence on copy number. This region (inc), which contains several repeated motifs, was also able to confer a degree of incompatibility when cloned into an otherwise unrelated mycobacterial replicon. pAL5000-derived plasmids carrying two copies of the inc region had a lower copy number and were less stable than the wild-type. These effects were only observed when the two regions were in the same orientation. Plasmids carrying only the inc region and no other parts of pAL5000 could be made to replicate if repA and repB were supplied in trans from another plasmid. Based on these findings, systems for selectively curing cells of one plasmid of a pair were designed and shown to be functional in Mycobacterium smegmatis. These have potential as a simple delivery system for achieving transposon mutagenesis or gene replacement in mycobacteria.
\end{abstract}

Keywords : origin activation, replication protein, curing of plasmids, copy number regulation, plasmid stability

\section{INTRODUCTION}

Introducing recombinant DNA into mycobacteria has been possible for several years, using Escherichia colimycobacteria plasmid shuttle vectors. Several replicons have been described and used in chimeric constructs (Snapper et al., 1988; Ranes et al., 1990; Stover et al., 1991; David et al., 1992; Qin et al., 1994; Beggs et al., 1995). Even so, little is known about plasmid replication and stability in mycobacteria; efficient shuttle vectors have largely been constructed by trial and error. Plasmid delivery systems for such essential genetic tools as transposon mutagenesis and gene replacement are also lacking. A temperature-sensitive plasmid has been described (Guilhot et al., 1992; Gavigan et al., 1995); although it can be employed in Mycobacterium smegmatis, it is less useful in the Mycobacterium tuberculosis complex, owing to the narrow temperature range of these organisms. Clearly, a greater understanding of the biology of

Abbreviations: LF, loss factor; SCR, single-cell resistance. mycobacterial plasmids would aid in carrying out genetic experiments.

The present work is a study of the regions and mechanisms governing the replication, compatibility and maintenance of the Mycobacterium fortuitum plasmid pAL5000 (Labidi $e t$ al., 1985), a widely used mycobacterial replicon. Two reports of the pAL5000 nucleotide sequence have been published (Rauzier et al., 1988; Labidi et al., 1992; GenBank accession nos M23557 and M60875, respectively) which are not identical and which predict five and two ORFs, respectively. Thus there is a need to study this replicon in greater detail.

The model organism for the work was $M$. smegmatis strain $\mathrm{mc}^{2} 155$ (Snapper et al., 1990), because of its fast growth and ease of manipulation. Through deletion mutagenesis and subcloning we have defined two ORFs, both of which seem to be necessary for replication and which act in trans. We have also defined a 435 bp region on pAL5000 which is needed in cis for replication and which influences copy number and incompatibility characteristics. In addition, we also determined changes in relative copy numbers arising from changes in the plasmid structure. 
The conclusions reached were subsequently evaluated in Mycobacterium bovis BCG and found to be valid in that organism; thus the replication mechanism of pAL5000 is similar in slow-growing as well as fast-growing mycobacteria, which has relevance in the context of establishing genetic systems for manipulating the pathogen $M$. $t u$ berculosis.

\section{METHODS}

Bacterial strains and media. E. coli strain $\mathrm{DH} 5 \alpha$ (Hanahan et $a l ., 1991)$ was used throughout to manipulate plasmid DNA. $E$. coli cells were grown in TY medium ( $16 \mathrm{~g}$ tryptone; $10 \mathrm{~g}$ yeast extract; $\left.\mathrm{l}^{-1}\right)$ with or without the addition of kanamycin $(\mathrm{Km}$; $\left.50 \mu \mathrm{g} \mathrm{ml}^{-1}\right)$, ampicillin $\left(50 \mu \mathrm{g} \mathrm{ml}^{-1}\right)$ or chloramphenicol $(\mathrm{Cm}$; $40 \mu \mathrm{g} \mathrm{ml}^{-1}$ ). M. smegmatis strain $\mathrm{mc}^{2} 155$ (Snapper et al., 1990) was grown in Lemco medium (Difco) or on Lemco agar plates. $M$. bovis BCG strain Pasteur was grown in Middlebrook $7 \mathrm{H} 9$ liquid medium (Difco) or on 7H10 agar (Difco) supplemented with oleic acid albumin dextrose (OADC; Difco).

PCR reactions. PCR primers were synthesized using a Pharmacia Gene Assembler Plus automated DNA synthesizer; sequences are shown in Table 1. Pfu DNA polymerase was purchased from Stratagene. The PCR products were cloned following digestion

Table 1. Sequences of the primers used in the PCR reactions

\begin{tabular}{|llc|}
\hline Oligonucleotide & \multicolumn{1}{c|}{ Sequence } & $\begin{array}{c}\text { Position in the pAL5000 } \\
\text { sequence (nt) }\end{array}$ \\
\hline$\Delta$ SL & TAACCGATCGATGCGCTGCACCAAC & $4039-4063$ \\
ORF2B & CGACACCGGATCCCCAATTGCGTA & $1100-1076$ \\
ORF1A & CACCAGCTCTAGAAGTTCGGGCGCTG & $4315-4340$ \\
ORF1B1 & GAGTGTGGATCCGTGGGGTGGCCCCTCA & $4537-4564$ \\
ORF1E & CGCGATATCGAGCCGAGAAC & $3872-3890$ \\
ORF1B & GCTGCCGGTTCTAGACGTCGCTGTAG & $767-742$ \\
OYUB10 & GCTGGTCTGCAGGACTCACCCGGTTG & $4158-4173$ \\
\hline
\end{tabular}

Table 2. DNA constructs used in the investigations described in this work

The numbering (nucleotides) refers to the pAL5000 sequence from Rauzier et al. (1988) (GenBank accession number M23557).

\begin{tabular}{|c|c|}
\hline Plasmid & Characteristics \\
\hline pUH4 & pYUB12 $\Delta \mathrm{Km}^{\mathrm{R}}(\operatorname{Tn} 5) \mathrm{Hy}^{\mathrm{R}}$ \\
\hline pUH10 & pYUB12 with extra iteron region $2(477-1627)$ in $H p a I$ site \\
\hline pUH11 & pUH4 with $A s c$ I deletion (152-1687) $\Delta \mathrm{ORF} 1 / 2$ \\
\hline pUH12 & pYUB12 with $A s c$ I deletion $(152-1687) \triangle O R F 1 / 2$ \\
\hline pUH19 & pAL5000 SmaI fragment $(3861-4837)$ in pUC18 \\
\hline pUH22 & pYUB12 $\Delta \mathrm{Km}^{\mathrm{R}}$ \\
\hline PUH25 & $\begin{array}{l}\text { pYUB12 with extra inc as } 1 \mathrm{~kb} \text { fragment }(3861-4837) \text { in EcoRV site in the same } \\
\text { orientation as wild-type inc }\end{array}$ \\
\hline pUH27 & pBL515 with iteron region 1 (3861-4837) from pAL5000 blunt in EcoRI site \\
\hline pUH29 & pAL5000 region $4325-752$ in pUC18; $\mathrm{Km}^{\mathrm{R}}(\operatorname{Tn} 903)$ \\
\hline pUH33 & $\begin{array}{l}\text { pYUB12 with extra inc as } 760 \text { bp fragment }(3861-4620) \text { in EcoRV site in the same } \\
\text { orientation as wild-type inc }\end{array}$ \\
\hline pUH34 & $\begin{array}{l}\text { pYUB12 with extra inc as } 760 \text { bp fragment }(3861-4620) \text { in EcoRV site in the } \\
\text { opposite orientation to wild-type inc }\end{array}$ \\
\hline pUH36 & pAL5000 region $3875-752$ in pUC18; $\mathrm{Km}^{\mathrm{R}}(\operatorname{Tn} 903)$ \\
\hline pUH51 & $\begin{array}{l}\text { pYUB12 with extra inc as } 440 \text { bp fragment }(4185-4620) \text { in EcoRV site in the same } \\
\text { orientation as wild-type inc }\end{array}$ \\
\hline pUH52 & pAL5000 region $4325-1093$ in pUC18; $\mathrm{Km}^{\mathrm{R}}(\operatorname{Tn} 903)$ \\
\hline pUH61 & pAL5000 region $3875-1093$ in pUC18; $\mathrm{Km}^{\mathrm{R}}(\operatorname{Tn} 903)$ \\
\hline pUH67 & pAL5000 EcoRV-HpaI fragment $(3876-1627)$ in pUC18; $\mathrm{Km}^{\mathrm{R}}(\operatorname{Tn} 903)$ \\
\hline $\mathrm{pUH} 70$ & pYUB12 with $\mathrm{Km}^{\mathrm{R}}$ from $\mathrm{Tn} 5$ replaced by $\mathrm{Km}^{\mathbf{R}}$ from $\mathrm{Tn} 903$ \\
\hline $\mathrm{pUH} 74$ & pAL5000 SacII-HpaI fragment $\left(3638-1627\right.$ in pUC18; $\mathrm{Km}^{\mathrm{R}}(\operatorname{Tn} 903)$ \\
\hline pUH77 & pAL5000 region $3861-4837$ in pUC18; $\mathrm{Km}^{\mathrm{R}}(\operatorname{Tn} 903)$ \\
\hline pUH83 & pAL5000 region $4050-1093$ in pUC18; $\mathrm{Km}^{\mathrm{R}}(\operatorname{Tn} 903)$ \\
\hline pUH93 & pAL5000 760 bp fragment inc $(3861-4620)$ in pUC18; $\mathrm{Km}^{\mathrm{R}}(\operatorname{Tn} 903)$ \\
\hline pUH96 & pAL5000 region $4165-1093$ in pUC18; $\mathrm{Km}^{\mathrm{R}}(\mathrm{Tn} 903)$ \\
\hline
\end{tabular}


with restriction enzymes at sites introduced by the primers. The different DNA constructs created for this study are shown in Table 2. DNA sequencing was carried out with the Sequenase kit (USB) utilizing the method of Sanger et al. (1977). The Wisconsin Genetics Computer Group package version 8 (Madison, Wisconsin, USA) was used to analyse DNA sequence data.

DNA extraction. Plasmid DNA was isolated from E. coli cells by standard procedures (Sambrook et al., 1989). For large-scale plasmid preparations, Wizard midipreps (Promega) were used. Mycobacterial plasmid DNA was extracted from $10 \mathrm{ml}$ cultures by treating harvested cells with lipase (Sigma; $1 \mathrm{mg} \mathrm{ml}^{-1}$ ) and lysozyme (Sigma; $1 \mathrm{mg} \mathrm{ml}^{-1}$ ) for $3 \mathrm{~h}$, followed by an alkaline lysis procedure similar to that used for E. coli. The lysates were treated repeatedly with phenol/chloroform and the DNA was precipitated with ethanol. Southern blots were carried out as descibed in Sambrook et al. (1989); DNA probes were labelled with $\left[{ }^{32} \mathrm{P}\right] \mathrm{dCTP}$. When detecting ssDNA, the denaturation step prior to transfer to nitrocellulose was omitted.

Electroporation. Competent $M$. smegmatis cells were prepared as described by Snapper et al. (1990). Transformation was performed on $300 \mu \mathrm{l}$ aliquots of cells with $1 \mu \mathrm{g}$ DNA. Transformation efficiency was measured for each batch of competent cells to minimize differences in electrocompetence. Transformation of $M$. bovis BCG was carried out as described by Dellagostin et al. (1993).

Copy number determination. Relative plasmid copy numbers were determined as single-cell resistance (SCR) to Km (Nordström, 1993). Cells were grown to late-exponential phase and $10^{5}$-fold dilutions were spread onto LB plates containing different concentrations of $\mathrm{Km}$. Total viable cell counts were also determined on LB plates with no selective pressure. The minimum concentration of $\mathrm{Km}$ necessary to inhibit cell growth was taken as the SCR.

Stability of plasmids. M. smegmatis cells carrying different $\mathrm{Km}^{\mathrm{R}}$ plasmids were grown in Lemco medium without selection for up to 120 generations and $10^{6}$-fold dilutions were made from each culture at stationary phase. The cells were plated on Lemco agar plates with and without selection and the fraction of resistant cells was taken as a measure of plasmid-carrying cells.

The loss factor (LF; Nordström \& Austin, 1989) was calculated according to the formula:

$\mathrm{LF}=1-\left(V_{1} / V_{2}\right)^{1 /\left(g_{1}-g_{2}\right)}$

where $V$ is the fraction of plasmid-carrying cells after $g$ generations.

\section{RESULTS AND DISCUSSION}

The shuttle plasmid pYUB12 (Snapper et al., 1988) was our starting point for studying pAL5000 propagation mechanisms in M. smegmatis. This construct is pAL5000, together with an E. coli origin of replication and the $\mathrm{Km}^{\mathrm{R}}$ gene from transposon $\mathrm{Tn} 5$. Removing the $\mathrm{Km}^{\mathrm{R}}$ gene by cutting with $B c l I$ and replacing it with the hygromycin (Hy)-resistance gene from Streptomyces bygroscopicus (Lydiate et al., 1989) on a 1739 bp BglII fragment yielded pUH4. Thus pYUB12 and pUH4 are identical except for the resistance conferred (Fig. 1).

Plasmids pYUB12 and pUH4 were electroporated into $M$. smegmatis either separately or together. The co-transformation efficiency was about $20 \%$ of that of each plasmid alone (Table 3), which indicated that the two
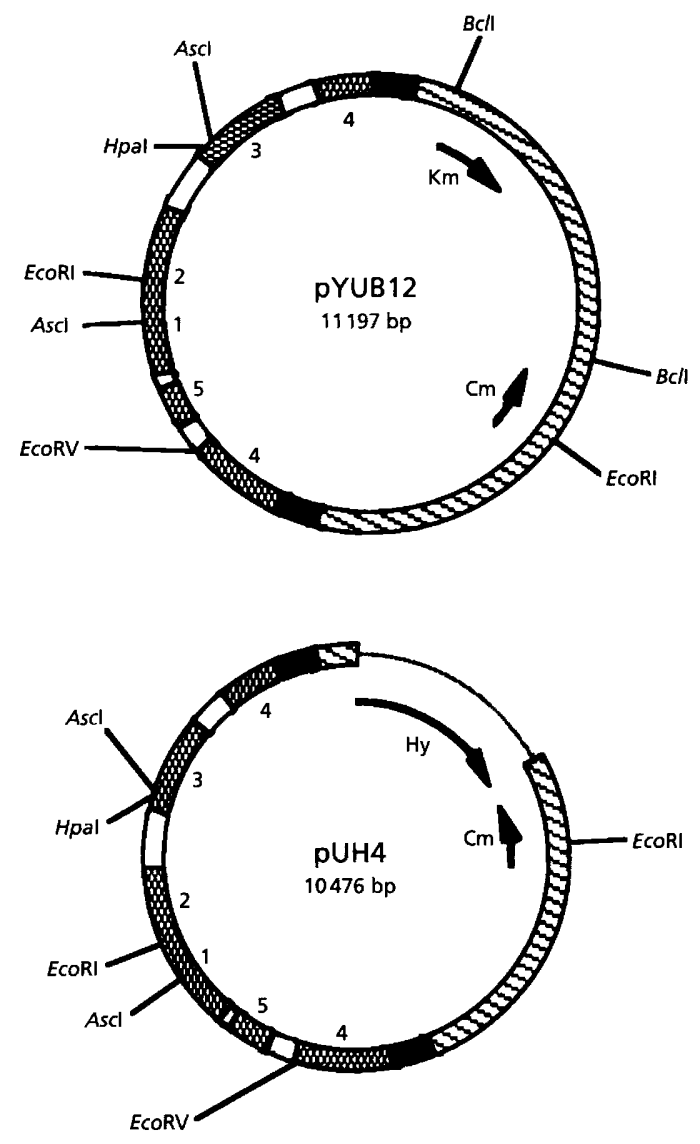

Fig. 1. Schematic map of the plasmids pYUB 12 and pUH4 used as standards in this work. The five ORFs on pAL5000 are shown as cross-hatched boxes (ORF4 was split by the cloning procedure). ORF1 and ORF2 overlap by $1 \mathrm{bp}$.

constructs could co-exist in the cells, suggesting room for further manipulation of their characteristics.

\section{Replication proteins}

There is one region on the pAL5000 DNA, according to the sequence reported by Rauzier et al. (1988), which comprises two ORFs flanked by sets of repeated sequences (Fig. 2). We have checked the pAL5000 sequence of the regions coding for ORF1 and ORF2 where the reports by Labidi et al. (1992) differ from that by Rauzier et al. (1988) and found no errors in that reported by Rauzier et al. (1988). We therefore refer to this as the correct sequence (GenBank accession no. M23557) throughout this work. The structural arrangement of the ORFs and iterons is very reminiscent of that of several E. coli plasmids, where the ORFs in such arrangements encode replication protein(s) and the repeated sequences (iterons) can be binding sites for the replication protein or serve as incompatibility determinants (Tsutsui et al., 1983; Churchward et al., 1983; Abeles et al., 1984; Smith \& Thomas, 1984; Kamio et al., 1984).

To test whether this region encodes proteins necessary for replication, the entire ORF2 and half of ORF1 were 
Table 3. Co-transformation efficiency for different plasmids electroporated into $M$. smegmatis together with pUH4

The co-transformation efficiency is the ratio of the transformation efficiency of the listed plasmid when $1 \mu \mathrm{g}$ of its DNA was added alone or along with $1 \mu \mathrm{g}$ pUH4 DNA, taking the frequency of transformation alone as 100 . The arrows indicate the relative orientation of the inc segments. pUH4 is the plasmid pYUB12 with the $\mathrm{Km}^{\mathrm{R}}$ gene replaced by $\mathrm{Hy}^{\mathrm{R}}$.

\begin{tabular}{|lc|}
\hline Plasmid & Co-transformation efficiency \\
\hline pYUB12 & 20 \\
pUH25 (1 kb SmaI fragment nt 3861-4837 inc $\rightarrow \rightarrow$ ) & $0 \cdot 1$ \\
pUH33 (760 bp SmaI-AccI nt 3861-4620 inc $\rightarrow \rightarrow$ ) & $0 \cdot 1$ \\
pUH34 (760 bp SmaI-AccI nt 3861-4620 inc $\leftarrow \rightarrow$ ) & 20 \\
pUH51 (440 bp HinclI-Acc nt 4185-4620 inc $\rightarrow \rightarrow$ ) & $0 \cdot 1$ \\
pBL515 & 50 \\
pUH27 (pBL515+1 kb inc) & 5 \\
\hline
\end{tabular}

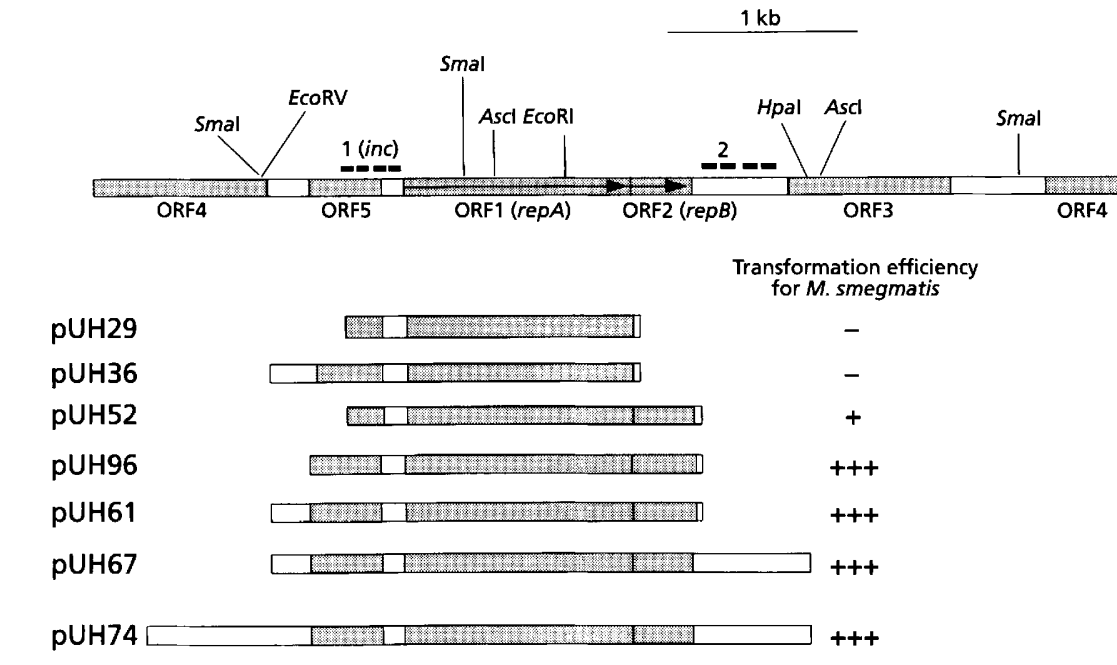

Fig. 2. Linear representation of pAL5000 in the form in which it is cloned in PYUB12, as well as of different pAL5000-derived (functional and non-functional) replicons studied in this work. The regions with a high frequency of repeated motifs are labelled 1 and 2 and are marked by dashed lines. ORF4 was split by the cloning procedure. The arrows indicate the orientations of ORF1 and ORF2. The transformation efficiency shown for $M$. smegmatis is not in absolute values. The lower value for pUH52 corresponds to the longer time necessary for transformants to grow after electroporation.

deleted by removing an $A s c \mathrm{I}$ fragment from pYUB12, yielding pUH12. We found that pUH12 was unable to replicate in $M$. smegmatis. However, in co-transformations with pUH4, double transformants were obtained. Both plasmids could be recovered intact from these transformants (not shown). Thus, the product(s) from the ORF1-ORF2 region are necessary for replication and act in trans. We found similarity between ORF1 and the Rep proteins from ColE2-related plasmids (Fig. 3), which has also been noted by Hiraga et al. (1994), a further indication that this protein has a role in replication. The ORF2 product has no significant similarities with any protein in the GenBank database.

Based on these findings, a set of potential replicons was constructed, using PCR to amplify distinct regions from pAL5000. The PCR products were cloned into pUC18 and the $\mathrm{Km}^{\mathrm{R}}$ gene from $\mathrm{Tn} 903$ was cloned into these constructs. The resulting plasmids were tested for their ability to replicate in $M$. smegmatis (Fig. 2).

Constructs containing ORF1 but not ORF2 (pUH29 and pUH36) were unable to replicate autonomously in $M$. smegmatis however much of the upstream sequences were included. If ORF2 was included, only a few hundred base pairs upstream of ORF1 were necessary for replication. The upstream region with the high incidence of repeats (region 1 in Fig. 2) was always necessary. The products from ORF1 and ORF2 were designated RepA (ORF1) and RepB (ORF2), respectively. The requirement for RepA and RepB corroborates the sequence reported by Rauzier et al. (1988), since the $\operatorname{rep} A$ and $r e p B$ ORFs are not present on the sequence published by Labidi et al. (1992).

Our results on their own do not show conclusively that RepA is necessary for replication at all, although it would be a great coincidence to have such high similarity to other replication proteins without a role for RepA. An indication that RepA is indeed necessary is the observation by McAdam et al. (1995) that a DraIII deletion of bp 62-615 (encoding the C-terminal end of RepA) creates a non-replicating plasmid, though the $\operatorname{rep} B$ gene would still be present on this construct.

The mycobacterial part of the commonly used shuttle vector pMV206 (Stover et al., 1991) consists of bp 43081364, which contains $20 \mathrm{bp}$ more upstream of ORF1 and $270 \mathrm{bp}$ more downstream of ORF2 than pUH52. This 


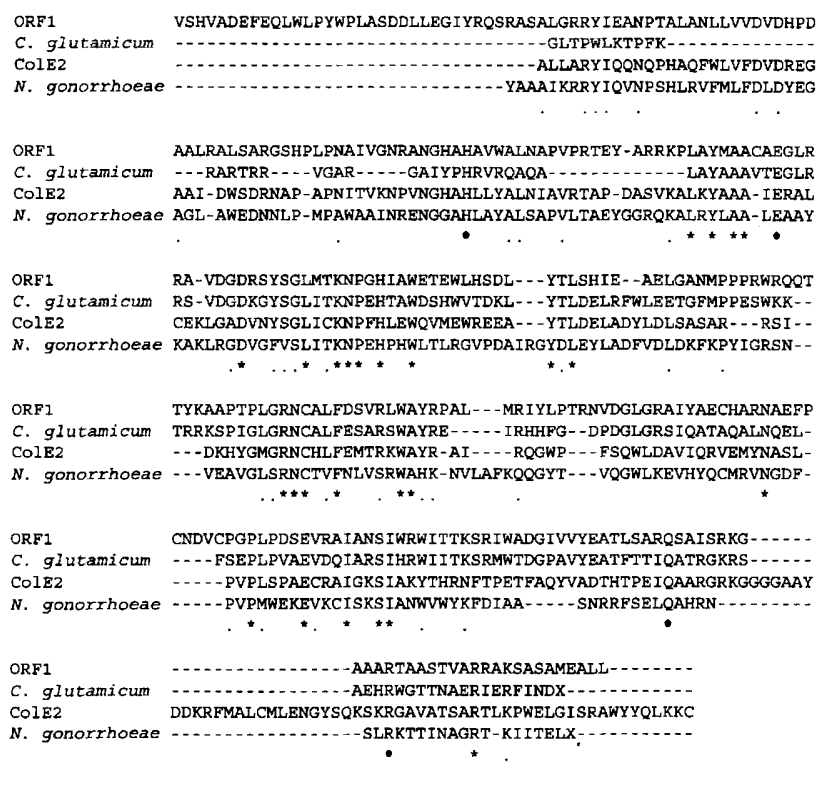

Fig. 3. Multiple sequence alignment of ORF1 to three replication proteins from ColE2-related plasmids. The sequence labelled ColE2 is a consensus sequence from eight $E$. coli ColE2related plasmid sequences in the GenBank database (accession nos D30057, D30058, D30056, D30055, D30059, D30062, D30063 and D30064). Asterisks under the sequence mark locations with identical amino acid residues in all sequences. Dots mark locations where an ORF1 residue is identical to two of the other sequences.

construct has been modified in an undefined way to remove restriction sites (Stover et al., 1991) and so comparisons with pUH52 are not straightforward.

The smallest functional replicons we constructed, pUH52 and pUH61, produced some ssDNA detectable by Southern blotting without previous denaturation of the DNA (not shown). If pAL5000 is similar to ColE2 plasmids, it would replicate by a $\Theta$-type mechanism and the presence of ssDNA in this context is somewhat surprising. These two vectors may have a use as delivery vectors in recombination experiments, since ssDNA is recombinogenic (West, 1992; Liu et al., 1993).

There was a difference in growth characteristics between cells harbouring replicons pUH52 and pUH61. The larger replicon, pUH61, which has an additional 450 bp upstream of ORF1, produced colonies after 3-4 d following transformation, whereas cells transformed with pUH52 took $5-6 \mathrm{~d}$ to grow on plates. There was no significant difference in transformation efficiency. Cells co-transformed with pUH52 and pUH4 took as long to form colonies as cells transformed with pUH52 only, indicating that the effect is not due to low expression of a replication protein.

\section{Copy number and incompatibility}

To test whether the repeated sequences on pAL5000 have a role in copy number control and incompatibility, we cloned additional copies of the regions (termed 1 and 2 in Fig. 2) into pYUB12. As a quick indication of incom-

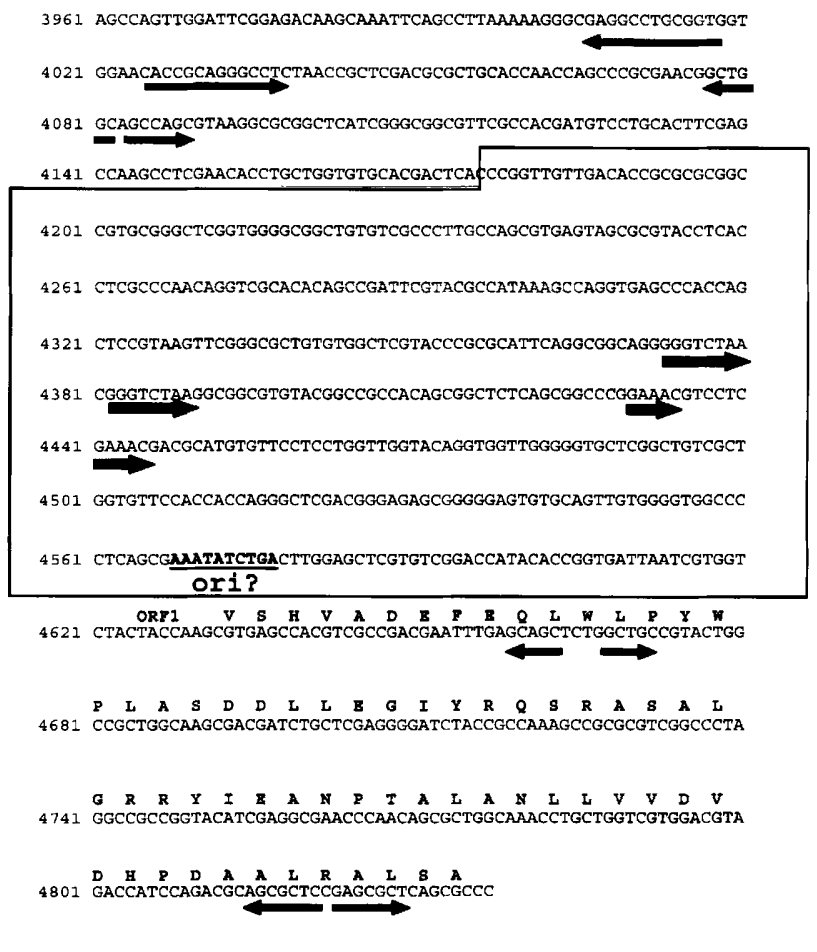

Fig. 4. DNA sequence of the inc region from pAL5000 (GenBank accession number M23557). The sequence starts $100 \mathrm{bp}$ downstream of the Smal site at nt 3862. The minimum defined inc region is boxed. Arrows mark direct or inverted repeats. All numbering refers to the sequence in GenBank M23557. The putative ori motif suggested by Rauzier et al. (1988) is shown underlined. The start of ORF1 translation has not been defined experimentally; that given here is used in the alignments in this work.

patibility, we scored for differences in co-transformation efficiency when the constructs were electroporated into $M$. smegmatis together with pUH4. This method is less conclusive than the standard assessment of incompatibility through displacement of a resident plasmid, but we thought it adequate for indications of whether the manipulated regions had an in vivo effect or not.

Doubling the number of iterons in region 2 (clone pUH10) had no noticeable effect on the co-transformation ability or stability of the plasmid. However, when region 1 was inserted as an additional $1 \mathrm{~kb} S \mathrm{maI}$ fragment (clone pUH25), a marked decrease in co-transformation efficiency was seen, with the value reduced almost to zero (Table 3). The transformation efficiency for each pYUB12-derived construct was not noticeably different from that of pYUB12. Hence, differences in co-transformation ability were ascribed to the effects of region 1 , which was designated inc.

The nucleotide sequence of this region is shown in Fig. 4. There are several regions of direct and inverted repeats in the sequence. Following subcloning, the active region was further narrowed down to the 435 bp region shown boxed in Fig. 4. This region was also present on all the functional replicons defined above and thus necessary for the replication of pAL5000. The decreased co-trans- 


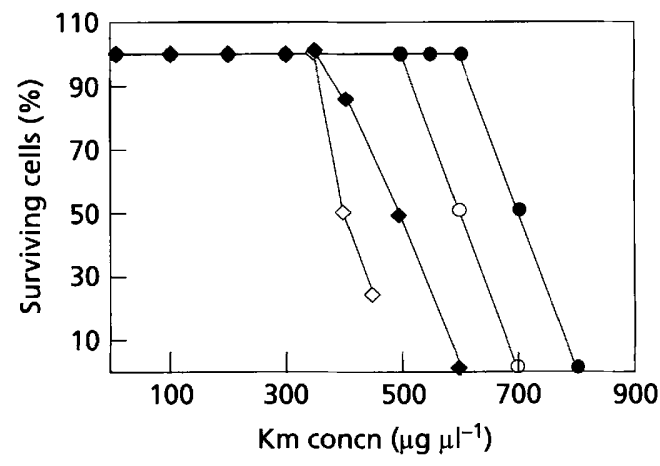

Fig. 5. SCR for several constructs shown as percentage of surviving cells under different $\mathrm{Km}$ concentrations. The experiments were repeated three times with consistent results; the graphs show the outcome of a typical experiment. $\diamond$, pUH25; O, pUH10; 0 , pYUB12; $\diamond$, pYUB12 co-existing with pUH4.

formation efficiency was only seen when the iterated regions were present in the same orientation (Table 3); plasmids with an extra inc in the opposite orientation behaved like wild-type. This argues for the sets of direct repeats in the region being responsible for the observed effects, since inverted repeats would be orientationindependent. There are two sets of direct repeats in the region (Fig. 4). One set has the repeats separated by 11 nucleotides, which would place them on the same side of the DNA helix, making them a possible binding site for a protein.

Incompatibility arises from the competition of two replicons for stable inheritance. To test whether the effects from the inc region of pAL5000 can be conferred to otherwise unrelated replicons, a construct ( $\mathrm{pUH} 27$ ) was generated by inserting the $1 \mathrm{~kb} S \mathrm{maI}$ fragment into the unrelated mycobacterium-E. coli shuttle vector pBL515 (David et al., 1992). Whilst the pair pBL515 and pUH4 was readily compatible, the co-transformation efficiency dropped by a factor of 10 when pUH27 and pUH4 were mixed (Table 3).

If the inc region is a cis-acting plasmid-specific sequence necessary for replication, each copy of inc would have the same effect as one copy of the whole plasmid in competing for components of the replication machinery of the cells. In that case, doubling the number of inc regions on one plasmid would reduce its copy number. We determined relative copy numbers as SCR to $\mathrm{Km}$, a method which has been shown to work well for low- and medium-copynumber plasmids (Nordström, 1993).

The SCRs for cells containing constructs pYUB12, pUH10 and pUH25 as well as for pYUB12 co-existing with pUH4, are shown in Fig. 5. The relative copy number for pYUB12 was set to 1 . When pUH4 was present, the SCR for cells carrying pYUB12 was reduced to $0 \cdot 65$, almost half that of cells carrying pYUB12 alone. This argues for the applicability of the method, since such a reduction in copy number was to be expected when two derivatives from the same replicon co-exist in one cell.

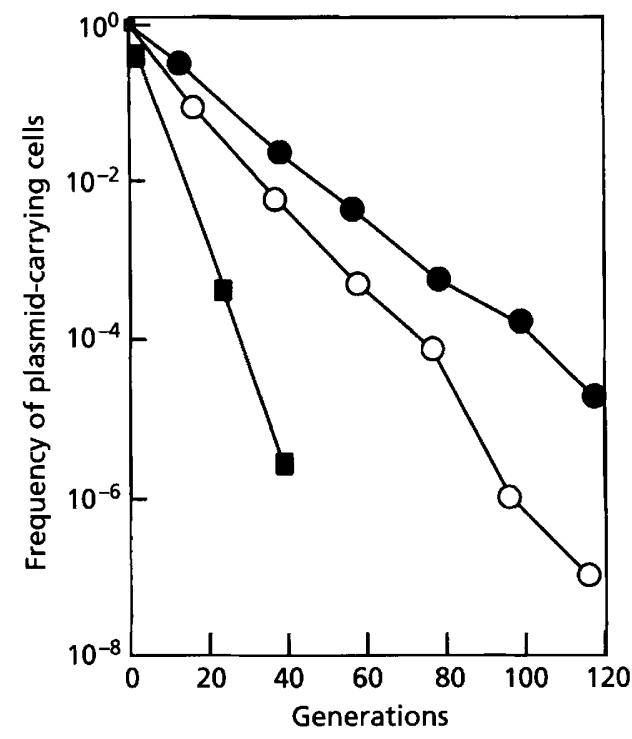

Fig. 6. Plasmid loss from populations of cells carrying different constructs with $\mathrm{Km}^{\mathrm{R}}$ genes. The experiments were done in duplicate with consistent results; the graphs show the outcome of a typical experiment. Cells carrying pYUB12 grown without selection; $O$, cells carrying pUH25, no selection; cells carrying pYUB12 and pUH4 under Hy selection for pUH4.

There was a decrease in SCR for pUH25 (which carries two copies of inc) compared with pYUB12, corresponding to a relative copy number of $0 \cdot 6$, or about half that of the wild-type. Thus the number of repeats in the inc region influences the plasmid copy number. In contrast, the plasmid pUH10, with twice the number of iterons in region 2 (Fig. 2), had a relative copy number of $0 \cdot 8$, only a slight decrease from that of wild-type. 'Thus there was a correlation between our co-transformation findings and the copy number experiments.

In determining the SCR, we noticed a difference between the level of resistance conferred by the $\mathrm{Km}^{\mathrm{R}}$ genes from transposons $\operatorname{Tn} 903$ and $\operatorname{Tn} 5$. The $\operatorname{Tn} 5 \mathrm{Km}^{\mathrm{R}}$ gene originates from the E. coli plasmid JR67 (Berg et al., 1975) while the $\mathrm{Km}^{\mathbf{R}}$ gene from Tn903 originates from the $E$. coli plasmid R6-5 (Berg et al., 1978; Nomura et al., 1978). To quantify the differences, the $\operatorname{Tn} 5 \mathrm{Km}^{\mathrm{R}}$ gene of pYUB12 was replaced by the $\mathrm{Km}^{\mathbf{R}}$ gene from $\mathrm{Tn} 903$, creating $\mathrm{pUH} 70$. In testing for SCR, pYUB12 scored approximately three times higher than pUH70. Hence, for the determination of relative copy number, only constructs with a common source of $\mathrm{Km}^{\mathrm{R}}$ genes were compared. There was no other difference between pYUB12 and pUH70, either in stability on their own or in loss rate when co-existing with pUH4, indicating that the two different $\mathrm{Km}^{\mathbf{R}}$ genes pose a similar burden on the cells.

If the construct $\mathrm{pUH} 25$ has half the copy number of the wild-type plasmid, this construct might also be expected to be less stable. The stability of the plasmids pYUB12 and $\mathrm{pUH} 25$ was monitored and the resulting curve is shown in Fig. 6. There was indeed a difference in stability 
between the two constructs; it translates into an LF (Nordström \& Austin, 1989) of $0 \cdot 12$ per generation of cells carrying pUH25, compared with 0.09 for cells harbouring the wild-type pYUB12. Both plasmids are considerably less stable than pUH4 (not shown). Since the mycobacterial parts of pYUB12 and pUH4 are identical and the only difference between these constructs is the resistance marker, the lower stability of pYUB12 compared with pUH4 indicates that the $\mathrm{Km}^{\mathrm{R}}$ gene is a greater burden on the cells than the $\mathrm{Hy}^{\mathrm{R}}$ gene.

The plasmid LF may be used to estimate copy number of plasmids (Nordström \& Austin, 1989). Given the relation $\mathrm{LF} \cong 2^{-2 n}$, this would give a copy number of $1 \cdot 7$ for pYUB12 and 1.5 for pUH25. However, these calculations from plasmid loss hold true only when the plasmids are segregated at random on cell division and there is no difference in growth rate between plasmid-carrying and plasmid-free bacteria (Nordström, 1993). If the $\mathrm{Km}^{\mathrm{R}}$ gene is indeed a burden on the cells, pYUB12 would be lost sooner than in the ideal case, leading to a calculated copy number which is lower than is actually the case. The greater stability of $\mathrm{Hy}^{\mathrm{R}}$-conferring plasmids does not imply a higher copy number, only a lower apparent loss rate. Nevertheless, all our investigations point to very low copy numbers for these mycobacterial plasmids and they corroborate the findings that doubling the inc region reduces the copy number.

\section{Origin activation}

If the replication proteins act in trans and the target is the inc region, then this part of pAL5000 may also be the origin of replication. To test this, we cloned the $1 \mathrm{~kb} S \mathrm{maI}$ fragment containing inc in pUC18 and added the $\mathrm{Km}^{\mathrm{R}}$ gene from $\mathrm{Tn} 903$. The resulting construct, $\mathrm{pUH77}$, was unable to replicate autonomously in $M$. smegmatis, but could be activated by co-transforming with pUH4. The same experiment was carried out with the $760 \mathrm{bp}$ inc fragment (construct pUH93; Table 2). Both constructs replicated in $M$. smegmatis when ORF1 and ORF2 were present in the cells on pUH4, although the transformants typically needed $2 \mathrm{~d}$ longer to grow on plates upon electroporation than did cells transformed with the pair pUH11 and pYUB12. The plasmids were recoverable from the transformants and we did not notice any recombination events between them.

Thus, all cis-acting elements necessary for replication are encoded on the region between bases 3861 and 4620 .

\section{Applications}

Incompatibility can be used selectively to cure a transformed mycobacterium cell of a plasmid. The pAL5000derived pair pYUB12/pUH4 are incompatible to such a degree that pYUB12 could be displaced by pUH4 under Hy selection over some 40 generations (Fig. 6). To test the feasibility of curing through incompatibility, pUH22 was created, a construct identical to pYUB12 but lacking the $\mathrm{Km}^{\mathrm{R}}$ gene. With pUH22 as a helper plasmid, it was possible for pUH11 and pUH12 to replicate in $M$.
Table 4. Plasmid loss from cells carrying the replicating plasmid pUH22, which has no resistance marker, together with the conditionally replicating pUH11 $\left(\mathrm{Hy}^{\mathrm{R}}\right)$ or pUH12 $\left(\mathrm{Km}^{\mathrm{R}}\right)$

\begin{tabular}{|lcc|}
\hline Plasmid pair* & $\begin{array}{c}\text { Total number } \\
\text { of cells } \dagger\end{array}$ & Resistant cells \\
\hline pUH12 $\left(\Delta r e p A ; \mathrm{Km}^{\mathrm{R}}\right)$ & & \\
$+\mathrm{pUH} 22$ & & $\mathbf{K m}^{\mathbf{R}}$ \\
1 & $1.5 \times 10^{7}$ & 0 \\
2 & $5.0 \times 10^{7}$ & 0 \\
3 & $1.0 \times 10^{7}$ & $2 \times 10^{5}$ \\
pUH11 $\left(\Delta r e p A ; \mathrm{Hy}^{\mathrm{R}}\right)$ & & \\
$+\mathrm{pUH} 22$ & $1.0 \times 10^{7}$ & 0 \\
1 & $1.0 \times 10^{7}$ & 0 \\
2 & $3.0 \times 10^{7}$ & 0 \\
3 & & $\mathbf{H y}^{\mathbf{R}}$ \\
pUH4+pYUB12 & $4.0 \times 10^{\mathbf{R}}$ & $4.0 \times 10^{7}$ \\
1 & $3.6 \times 10^{7}$ & $2.8 \times 10^{7}$ \\
2 & $3.0 \times 10^{7}$ & $2.8 \times 10^{7}$ \\
3 & & \\
\hline
\end{tabular}

* Cells were picked from plates from transformations with the plasmid pairs $\mathrm{pUH} 22 / \mathrm{pUH} 11$ or $\mathrm{pUH} 22 / \mathrm{pUH} 12$ and were plated without selection. Single colonies grown up on these plates were subsequently titrated onto selective and non-selective plates to determine the fraction of antibiotic-resistant and thus presumably plasmid-carrying cells in each colony. The pair pYUB12/pUH4, where both plasmids replicate, was used as a comparison with the pair $\mathrm{pUH} 12 / \mathrm{pUH} 22$; single colonies from these plates were titrated onto Hy plates to score for loss of pUH4.

t The total number of cells refers to the number of cells in each single colony picked from plates with no selection.

smegmatis. Selection for pUH11 or pUH12 was sufficient to keep pUH22 in the cells, since loss of the helper would lead to loss of the plasmid carrying resistance. The use of only one antibiotic marker to select for two plasmids is advantageous given the dearth of suitable markers for mycobacteria. Again we observed no recombination between plasmids over $20-50$ generations.

Transformants carrying pUH22 and either pUH11 or pUH12 were spread on plates with no selection and single colonies were allowed to form. These were then titrated onto selective plates to score for plasmid retention. The results (Table 4 ) show that the conditionally replicating plasmid with the antibiotic resistance was efficiently lost over the approximately 23 generations needed to form a single colony when the selection pressure was removed. Thus the conditionally replicating plasmid was lost more efficiently than replicating ones, as indicated by a comparison with the pair pYUB12/pUH4 (Table 4).

To test whether the conclusions drawn from work with $M$. smegmatis are valid for slow-growing mycobacteria, we have confirmed some of our findings in another host, $M$. bovis BCG. Co-transforming with the pair pYUB12 and pUH4 gave results similar to those obtained with $M$. smegmatis. pUH11 also did not replicate in $M$. bovis BCG but it was again possible to activate this replicon by 
supplying the Rep proteins in trans on pUH4. This indicates that the mechanisms of replication of pAL5000 are very similar in fast-growing and slow-growing mycobacteria. Thus the methods developed for $M$. smegmatis may well have their use in manipulating the pathogen $M$. tuberculosis.

\section{ACKNOWLEDGEMENTS}

This work was supported by the Glaxo-Wellcome Action TB Initiative. Thanks to Ken Duncan for critical reading of the manuscript. P. Stolt wishes especially to thank V. Mizrahi for stimulating discussions.

\section{REFERENCES}

Abeles, A. L., Snyder, K. M. \& Chattoraj, D. K. (1984). P1 plasmid replication: replicon structure. J Mol Biol 173, 307-324.

Beggs, M. L., Crawford, J. T. \& Eisenach, K. D. (1995). Isolation and sequencing of the replication region of Mycobacterium avium plasmid pLR7. J Bacteriol 177, 4836-4840.

Berg, D. E., Davies, J., Allet, B. \& Rochaix, J. D. (1975). Transposition of $\mathrm{R}$ factor genes to bacteriophage lambda. Proc Natl Acad Sci US A 72, 3628-3632.

Berg, D. E., Jorgensen, R. \& Davies, J. (1978). Transposable kanamycin-neomycin resistance determinants. In Microbiology-1978, pp. 13-15. Edited by D. Schlessinger. Washington, DC: American Society for Microbiology.

Churchward, G., Linder, P. \& Caro, L. (1983). The nucleotide sequence of replication and maintenance functions encoded by plasmid pSC101. Nucleic Acids Res 11, 5645-5659.

David, M., Lubinsky-Mink, S., Ben-Zvi, A., Ulitzur, S., Kuhn, J. \& Suissa, M. (1992). A stable Escherichia coli-Mycobacterium smegmatis plasmid shuttle vector containing the mycobacteriophage D29 origin. Plasmid 28, 267-271.

Dellagostin, O. A., Wall, S., Norman, E., O'Shaughnessy, T., Dale, J. W. \& McFadden, J. (1993). Construction and use of integrative vectors to express foreign genes in mycobacteria. Mol Microbiol 10 , 983-993.

Gavigan, J. A., Guilhot, C., Gicquel, B. \& Martin, C. (1995). Use of conjugative and thermosensitive cloning vectors for transposon delivery to Mycobacterium smegmatis. FEMS Microbiol Lett 127, 35-39.

Guilhot, C., Gicquel, B. \& Martin, C. (1992). Temperature-sensitive mutants of the Mycobacterium plasmid pAL5000. FEMS Microbiol Lett 98, 181-186.

Hanahan, D., Jessee, J. \& Bloom, F. R. (1991). Plasmid trans formation of Escherichia coli and other bacteria. Methods Enzymol 204, 63-114.

Hiraga, S. I., Sugiyama, T. \& Itoh, T. (1994). Comparative analysis of the replicon regions of eleven ColE2-related plasmids. $J$ Bacteriol 176, 7233-7243.

Kamio, Y., Tabuchi, A., Itoh, Y., Katagiri, H. \& Terawaki, Y. (1984). Complete nucleotide sequence of mini-Rts1 and its copy mutant. $J$ Bacteriol 158, 307-312

Labidi, A., David, H. L. \& Roulland-Dussoix, D. (1985). Cloning and expression of mycobacterial plasmid DNA in Escherichia coli. FEMS Microbiol Lett 30, 221-225.

Labidi, A., Mardis, E., Roe, B. A. \& Wallace, R. J., Jr (1992). Cloning and DNA sequence of the Mycobacterium fortuitum var. fortuitum plasmid pAL5000. Plasmid 27, 130-140.

Liu, S.-K., Eisen, J. A., Hanawalt, P. C. \& Tessman, I. (1993). rec.A mutations that reduce the constitutive coprotease activity of the RecA1202( $\left.\mathrm{Prt}^{\mathrm{C}}\right)$ protein: possible involvement of interfilament association in proteolytic and recombination activities. $J$ Bacteriol $175,6518-6529$.

Lydiate, D. J., Ashby, A. M., Henderson, D. J., Kieser, H. M. \& Hopwood, D. A. (1989). Physical and genetic characterization of chromosomal copies of the Streptomyces coelicolor mini-circle. $J$ Gen Microbiol 135, 941-955.

McAdam, R. A., Weisbrod, T. R., Martin, J., Scuderi, J. D., Brown, A. M., Cirillo, J. D., Bloom, B. R. \& Jacobs, W. R., Jr (1995). In vivo growth characteristics of leucine and methionine auxotrophic mutants of Mycobacterium bovis BCG generated by transposon mutagenesis. Infect Immun 63, 1004-1012.

Nomura, N., Yamagishi, H. \& Oka, A. (1978). Isolation and characterization of transducing coliphage fd carrying a kanamycin transposon. Gene 3, 39-51.

Nordström, K. (1993). Plasmid replication and maintenance. In Plasmids: a Practical Approach, 2nd edn, pp. 2-38. Edited by K. G. Hardy. Oxford: Oxford University Press.

Nordström, K. \& Austin, S. J. (1989). Mechanisms that contribute to the stable segregation of plasmids. Annu Rev Genet 23, 37-69.

Qin, M., Taniguchi, H. \& Mizuguchi, Y. (1994). Analysis of the replication region of a mycobacterial plasmid, pMSC262.J Bacteriol 176, $419-425$.

Ranes, M. G., Rauzier, J., Lagranderie, M., Gheorghiu, M. \& Gicquel, B. (1990). Functional analysis of pAL5000, a plasmid from Mycobacterium fortuitum: construction of a 'mini' mycobacteriumEscherichia coli shuttle vector. J Bacteriol 172, 2793-2797.

Rauzier, J., Moniz-Pereira, J. \& Gicquel-Sanzey, B. (1988). Complete nucleotide sequence of pAL5000, a plasmid from Mycobacterium fortuitum. Gene 71, 315-321.

Sambrook, J., Fritsch, E. F. \& Maniatis, T. (1989). Molecular Cloning: a Laboratory Manual, 2nd edn. Cold Spring Harbor, NY: Cold Spring Harbor Laboratory.

Sanger, F., Nicklen, S. \& Coulson, A. R. (1977). DNA sequencing with chain-terminating inhibitors. Proc Natl Acad Sci USA 74, 5463-5467.

Smith, C. A. \& Thomas, C. M. (1984). Nucleotide sequence of the $\operatorname{trf} A$ gene of broad host-range plasmid RK2. $J$ Mol Biol 175, 251-262.

Snapper, S. B., Lugosi, L., Jekkel, A., Melton, R. E., Kieser, T., Bloom, B. R. \& Jacobs, W. R., Jr (1988). Lysogeny and transformation in mycobacteria: stable expression of foreign genes. Proc Natl Acad Sci US A 85, 6987-6991.

Snapper, S. B., Melton, R. E., Mustafa, S., Kieser, T. \& Jacobs, W. R., Jr (1990). Isolation and characterization of efficient plasmid transformation mutants of Mycobacterium smegmatis. Mol Microbiol 4, 1911-1919.

Stover, C. K., de la Cruz, V. F., Fuerst, T. R., Burlein, J. E., Benson, L. A., Bennett, L. T., Bansal, G. P., Young, J. F., Lee, M. H., Hatfull, G. F., Snapper, S. B., Barletta, R. G., Jacobs, W. R., Jr \& Bloom, B. R. (1991). New use of BCG for recombinant vaccines. Nature 351, 456-460.

Tsutsui, H., Fujiyama, A., Murotsu, T. \& Matsubara, K. (1983). Role of nine repeating sequences of the mini-F genome for expression of F-specific incompatibility phenotype and copy number control. J Bacteriol 155, 337-344.

West, S. C. (1992). Enzymes and molecular mechanisms of genetic recombination. Annu Rev Biochem 61, 603-640.

Received 25 March 1996; revised 23 May 1996; accepted 28 May 1996. 